\title{
Utilização do software Geogebra no ensino de óptica geométrica de lentes esféricas
}

\author{
ANDré Luís Miranda de BARCEllos COElHo* \\ Secretaria de Educação do Distrito Federal, SEDF, Brasília, Brasil.
}

\begin{abstract}
Resumo
Neste trabalho compartilho uma proposta de utilização do software Geogebra para ensinar, de maneira mais significativa, óptica geométrica de lentes delgadas esféricas. Diversos trabalhos em ensino de ciências mostram que o uso de tecnologia em sala de aula pelos professores e estudantes favorece o engajamento dos partícipes do processo. Além disso, cria um ambiente mais propício para a aprendizagem significativa de uma gama maior de estudantes. Construí, para alcançar esse fim, uma proposta de trabalho para professores do segundo ano do ensino médio utilizando uma plataforma digital na qual é possível interagir com objetos geométricos compatíveis com a descrição de lentes esféricas delgadas. Acredito que a utilização desse recurso como abordagem complementar ao assunto pode contribuir positivamente para aprendizagem significativa dos estudantes
\end{abstract}

Palavras-chave: Uso de tecnologia na educação, Geogebra, Óptica Geométrica.

*prof.barcellos@hotmail.com 


\begin{abstract}
In this paper, I share a proposal to use Geogebra software to teach, in a more meaningful way, geometric optics of thin spherical lenses. Several works in science teaching show that the use of technology in the classroom by teachers and students favors the engagement of process participants. As well as creating a more conducive environment for meaningful learning for a wider range of students. To achieve this goal, I have constructed a proposal of work for teachers of high school using a digital platform in which it is possible to interact with geometric objects compatible with the description of thin spherical lenses. I believe that the use of this resource as a complementary approach to the subject can contribute positively to meaningful student learning.
\end{abstract}

Keywords: Use of technology in education, Geogebra, Geometric Optics.

\title{
1 Introdução
}

O ensino de física em geral esbarra em uma grande quantidade de desafios. Entre eles, podemos elencar a dicotomia da abordagem utilizada tradicionalmente para ensinar matemática e física no ensino médio e a falta de significado para o aluno na aprendizagem de fenômenos naturais. Sobre esse último, PIETROCOLA diz que "os conceitos nela [na Ciência] presentes são por demais abstratos, mantendo uma relação indireta com situações presentes no cotidiano" [1]. Atribui-se, frequentemente, ao fracasso no ensino de física a falta de domínio da linguagem matemática [1]. É flagrante que a matemática é parte integrante e indissociável da física. Entretanto, quando se trata do ensino de física para o ensino médio, muitos professores preferem uma abordagem puramente fenomenológica a enfrentar o desafio de ensinar também a linguagem física padrão. Esse tipo de abordagem acaba por estabelecer uma dicotomia, que não deveria existir, entre a abordagem didática conceitual e matemática.

Diversos autores $[2,3,4]$ abordam o problema da disparidade entre a ferramenta ou linguagem matemática e a sua funcionalidade para a descrição de fenômenos naturais. Em meu trabalho de mestrado, pude atestar, por exemplo, que meus alunos foram incapazes de utilizar o ferramental matemático, por suposto conhecido, quando se depararam com um fenômeno natural que carecia de interpretação lógica [5]. Desde então, me imbuí com o senso de explicitar, sempre que possível, a íntima relação que existe entre a lógica matemática e a fenomenologia natural.

Foi nesse espírito de investigação que me surgiu a ideia de utilizar uma ferramenta que meus colegas matemáticos utilizam em sala de aula frequentemente para ensinar geometria e adaptá-la para o ensino de óptica geométrica. A ferramenta em questão é o software Geogebra que, entre outras tantas funcionalidades, permite, mesmo a um estudante sem muito letramento científico, modelar diversos objetos geométricos. 
O segundo desafio mencionado, diz respeito à aprendizagem significativa de conceitos que podem soar vagos ou imediatamente inúteis àqueles não engajados na investigação natural. Esse é um desafio que envolve não só a comunidade de educadores, mas também - e, talvez, principalmente - o corpo discente da comunidade escolar. Para diversos teóricos e da aprendizagem (como Ausubel,Vygotsky e tantos outros), é necessário que haja certa sinergia entre educador e aprendiz para que aconteça a aprendizagem.

Neste cenário, há quem defenda que o uso de tecnologias pode ser um grande aliado para os educadores e até um deleite para os aprendizes $[6,7,8]$. Há algumas iniciativas educacionais que mostram resultados interessantes em relação à aprendizagem ser mais significativa do que no método tradicional de ensino, como o uso das simulações do PHeT proposta por Arantes [9] ou do Google Docs para a educação [10]. Para o ensino de óptica geométrica especificamente, o uso de tecnologias educacionais parecem favorecer o engajamento dos aprendizes [11].

O ensino oferecido em nível de Ensino Médio é tradicionalmente propedêutico e sem compromisso com as demandas imediatas dos alunos envolvidos no processo. Como consequência direta desse tipo de abordagem obtemos resultados medianos, com vistas do que se almeja alcançar com o ensino de física. Esses objetivos almejados são explicitados nos PCN para Ciências da Natureza, Matemática e suas Tecnologias, na página 6 , onde é dito:

"Os objetivos do Ensino Médio em cada área do conhecimento devem envolver, de forma combinada, o desenvolvimento de conhecimentos práticos, contextualizados, que respondam às necessidades da vida contemporânea, e o desenvolvimento de conhecimentos mais amplos e abstratos, que correspondam a uma cultura geral e a uma visão de mundo." [12]

Se essa maneira de ensinar que tradicionalmente é pouco prática e muito informativa não mudar, todo o processo está fadado a conduzir sempre aos mesmos resultados medianos que se observam nas últimas avaliações como o PISA [13], que revela que o desempenho em Ciências de nossos estudantes se mantém no mesmo patamar desde 2006, atrás de diversos países da América Latina, como México, Colômbia e Costa Rica [13].

Este trabalho utiliza o software Geogebra que é produto de um desses esforços para a melhoria da educação. Almeja-se aqui, oferecer uma possibilidade para o ensino de óptica geométrica de lentes esféricas delgadas, para professores de física do ensino médio, que possua, ao mesmo tempo, uma sólida construção matemática e que seja acessível aos estudantes desse nível de escolaridade

\section{O software Geogebra}

Em www.geogebra.org encontra-se uma descrição geral do que é o software Geogebra: "O GeoGebra é um software [livre de General Public License] de matemática dinâmica para todos os níveis de ensino que reúne Geometria, Álgebra, Planilha de Cálculo, Gráficos, Probabilidade, Estatística e Cálculos Simbólicos em um único pacote fácil de se usar [...]”. Neste endereço também é possível fazer o download gratuito desta ferramenta educacional vencedora de diversos prêmios como o MNU Award e o Multimedia Educational Resource for Learning and Online Teaching (segundo informações do site). 




Figura 1: Janela de diálogo inicial do software Geogebra.

O software oferece uma interface convidativa e, de certa forma, intuitiva para professores e estudantes interessados em explorar as mais diversas representações gráficas não apenas no que concerne à geometria propriamente, mas também a algumas ferramentas interessantes sobre o cálculo algébrico.

Por essas características inovadoras, o Geogebra é um dos softwares mais utilizados nas salas de aula de matemática e até em disciplinas correlatas, como a física. Diversos autores já escreveram sobre a utilização desse programa de maneiras criativas e interessantes $[14,15,16]$.

Das diversas ferramentas que ele oferece ao usuário utilizaremos neste trabalho aquelas relativas à geometria, com o propósito de recomendar o uso para o ensino de óptica geométrica na disciplina de física. Mais especificamente, no ensino do comportamento óptico de lentes delgadas.

Assim que o usuário executa o software abre-se uma caixa de diálogo com algumas opções (como ilustrado na Figura 1), entre elas uma chamada Geometria.

Com alguns cliques é possível construir, por exemplo, qualquer lente delgada esférica como é ilustrado na Figura 2. Os arquivos na extensão .ggb (extensão de arquivos produzidos pelo software Geogebra) podem ser encontrados no website criado para a divulgação desse trabalho e acessado pelo endereço https://sites.google.com/site/opticageogebra/. São seis arquivos ao todo, cada um descrevendo uma lente esférica delgada como as ilustradas na Figura 2.

\section{O comportamento óptico de lentes esféricas}

Em óptica geométrica utiliza-se o princípio de mínimo tempo (ou princípio de Fermat) para se obter a geometria mais simples possível para a modelagem do comportamento da luz em instrumentos ópticos. Este princípio enuncia que a trajetória seguida por um raio luminoso entre dois pontos é aquela em que percorrida por ele no menor intervalo de tempo possível. Como corolário desse princípio, temos a propagação retilínea da luz e, consequentemente, a lei da reflexão em espelhos planos .

Ainda como corolário desse princípio é possível obter a lei de Snell-Descartes para a refração, como demonstrado por Feynman [17]. A lei de Snell pode ser escrita da seguinte forma: 




Figura 2: Exemplos de lentes delgadas utilizando o Geogebra. As figuras a), b) e c) são representantes das chamadas lentes delgadas de extremidades finas. Já d), e) e f) são representantes das lentes delgadas de extremidades grossas.

$$
\frac{n_{1}}{n_{2}}=\frac{\sin (\hat{r})}{\sin (\hat{\imath})},
$$

onde $n_{1}$ é o índice de refração do meio onde se propaga o raio incidente, $n_{2}$ é o índice de refração do meio onde se propaga o raio refratado, $\hat{r}$ e $\hat{\imath}$ os ângulos de refração e incidência, respectivamente.

Deduz-se da equação (1) que quando $n_{1}>n_{2}$, o raio refratado se afasta da reta normal em relação à direção de propagação do raio incidente. E, logicamente, quando $n_{1}<n_{2}$, o comportamento contrário é observado.

No caso de lentes delgadas, o comportamento óptico de cada uma delas depende:

- do formato da lente - se suas bordas são finas ou grossas. Essa geometria, de fato, define as características dos raios de curvatura das faces da lente;

- do índice de refração relativo entre o meio e o material da lente. Como será demonstrado na próxima seção, utilizando-se a lei de Snell e geometria plana, é possível determinar o comportamento de uma lente delgada, não se restringindo, inclusive, às esféricas.

\section{Utilizando o Geogebra para o ensino do comportamento óptico de lentes esféricas}

O software Geogebra oferece uma gama interessante de possibilidades para explorar o ensino de óptica geométrica para o Ensino Médio, como demonstra a dissertação de SILVA [18]. Neste trabalho, evidencio um potencial uso desse software para o ensino do comportamento óptico de lentes esféricas delgadas. 


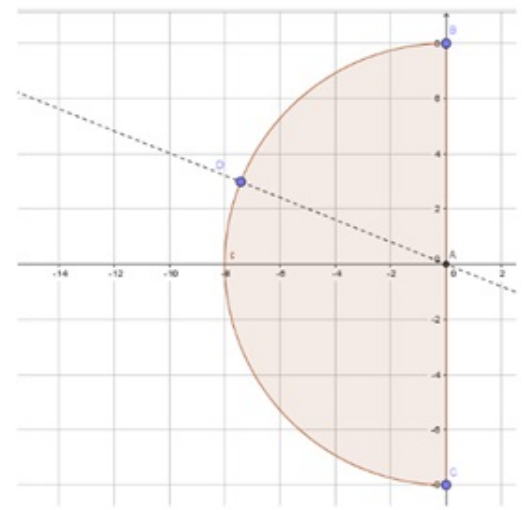

Figura 3: Representação de uma lente plano-convexa. O ponto A, representa o centro do semicírculo formado por essa lente esférica. O ponto D é o ponto onde incidirá um raio luminoso. A reta pontilhada que passa por A e D é normal à curvatura.

Na Figura 3, é representado um corte transversal de uma lente esférica do tipo plano-convexa. Por se tratar de uma lente esférica, esse corte é um semicírculo com centro em A. No ponto D, é representada uma reta normal à superfície curva da lente e é onde se incidirá um raio luminoso horizontal. Considera-se ainda que o meio cuja lente esteja imersa seja o ar e que o material de que é feito a lente é mais refringente que o ar.

Ao fazer incidir um raio luminoso horizontal (vetor $\mathrm{u}$ ) em $\mathrm{D}$, devemos considerar três comportamentos possíveis: reflexão, refração e absorção. O raio incidente (vetor u), refletido (vetor v, vide Figura 4(a)) e o raio refratado (vetor g, vide Figura 4(b)) são representados nas imagens a seguir e são facilmente verificáveis empiricamente. A parcela absorvida pelo material, por óbvio, não é representável nos termos escolhidos nas imagens .

$\mathrm{Na}$ outra face da lente incide o raio interno à lente representado pelo vetor g. Novamente, acontece refração, reflexão e absorção. Na Figura 5(a) está representado apenas o raio refratado que é o que nos interessa analisar neste trabalho.

Fazendo o mesmo no quadrante inferior do semicírculo observamos o claro comportamento convergente da lente analisada (ver 5(b)).

Se mudássemos a condição inicial do sistema e impuséssemos um índice de refração relativo entre o ar e a lente maior que 1, observaríamos que essa mesma lente seria divergente.

O software Geogebra, portanto, possibilita uma investigação do modelo geométrico para o comportamento óptico de uma lente esférica delgada sem a necessidade de visitar um laboratório didático (que em muitas instituiçõos de ensino básico sequer existe). Além disso, pode ser também uma ferramenta didática para validação teórica da lei de Snell. Neste trabalho não foi evidenciado, entretanto, ao definir dois segmentos de reta no software Geogebra é possível medir o ângulo entre eles (com duas casas decimais de precisão) e determinar, dessa forma, os ângulos de incidência e refração. Fazendo isso em diversos pontos ao longo do perímetro do semicírculo da face em que incidem os raios luminosos - aplicando para cada refração a lei de Snell apropriadamente - é possível investigar o comportamento óptico da lente com maior precisão. 

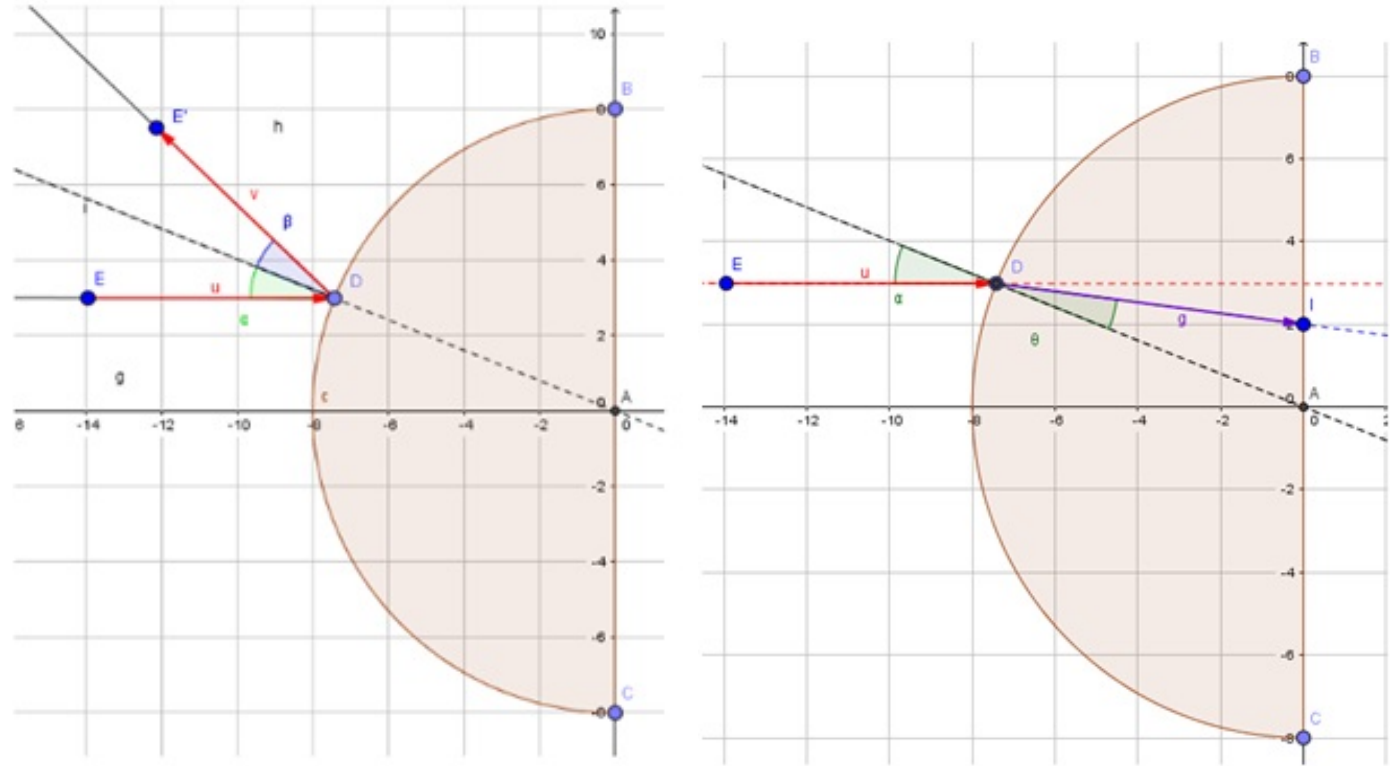

Figura 4: (a) Raio parcialmente refletido na interface ar-lente. $O$ vetor u representa o raio incidente no ponto $D$ que é contido na semicircunferência. O vetor $v$ representa o raio refletido. Observa-se que o ângulo de incidência $\alpha$ (em verde) é congruente ao ângulo de reflexão $\beta$, em azul. (b) Raio parcialmente refratado na interface ar-lente. $O$ vetor u representa o raio incidente no ponto $D$ que é contido na semicircunferência. $O$ vetor g representa o raio refratado. Observa-se, via lei de Snell, que o ângulo de incidência $\alpha$ maior que o ângulo de refração $\theta$.

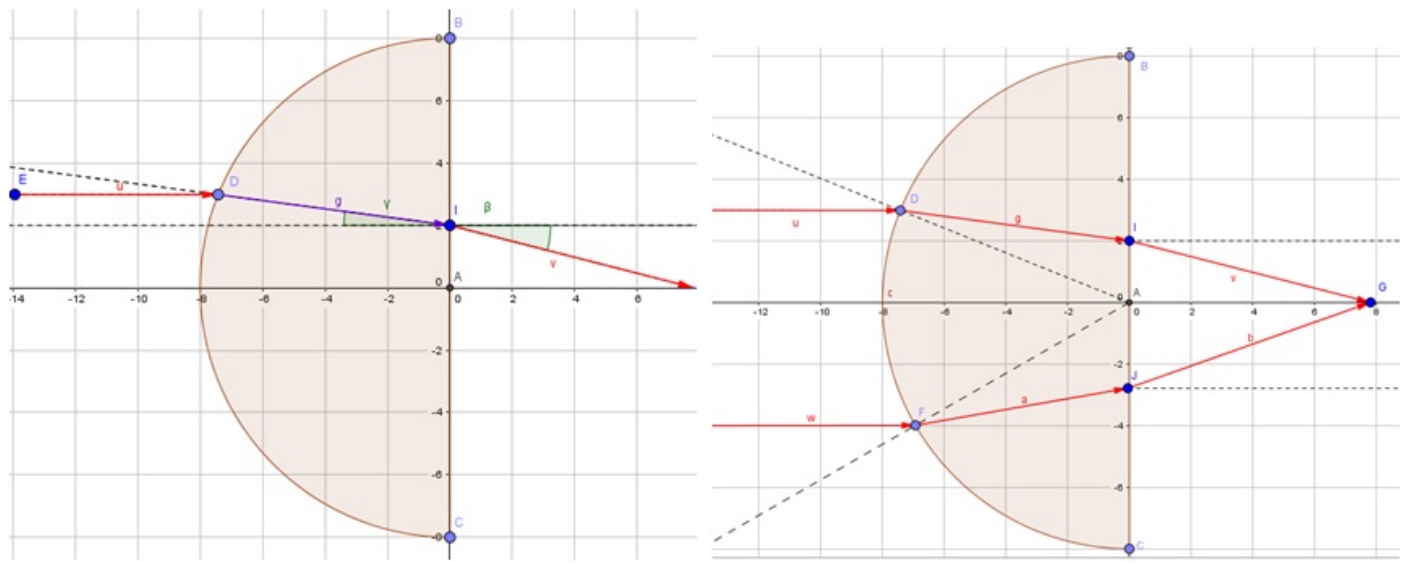

Figura 5: (a) Raio parcialmente refratado na interface lente-ar. O vetor g representa o raio incidente no ponto I que é contido na face plana da lente. O vetor h representa o raio refratado para o ar. Novamente via lei de Snell, observa-se que o ângulo de incidência $\gamma$ é menor que o ângulo de refração $\beta$. (b) Representação da refração de dois raios luminosos incidindo em dois pontos distintos na face curva de uma lente esférica do tipo plano-convexa. As retas pontilhadas representam as retas normais em cada um dos pontos de interface demarcados em azul na imagem. 


\section{Outros Exemplos}

Uma vez compreendido o método computacional sugerido neste trabalho, utilizando o software Geogebra, é possível extrapolá-lo para qualquer outra lente delgada, não se restringindo às esféricas. É possível, por exemplo, construir parábolas e hipérboles no software e atribuir retas perpendiculares a qualquer ponto contido nessas curvas e assim determinar o comportamento óptico de uma lente não esférica com certa facilidade.

No trabalho de SILVA [18], o autor cita diversos exemplos de uso do software Geogebra para determinar a reflexão em espelhos planos e esféricos, além de exemplos de aplicação da própria lei de Snell para dioptros planos e prismas. Encorajo a leitura dessa dissertação pela relevância do trabalho desenvolvido em relação ao uso do software Geogebra para o ensino de óptica geométrica.

Como última sugestão de trabalho, pode-se usar o software Geogebra para demonstrar (de diversas formas, inclusive) o princípio de Fermat. Uma leitura clara e que exemplifica uma abordagem intuitiva para essa demonstração figura nas Lições de Física de Richard Feynman, Volume 1, $26-3$ e 26-4 [17]. Nessa demonstração, utilizando alguns segmentos de retas coplanares, o autor demonstra que, para um espelho plano, o tempo mínimo entre dois pontos refletindo-se no meio do caminho é obtido quando os ângulos de incidência e reflexão são iguais.

\section{Conclusão}

Nas últimas décadas, os debates sobre o uso de tecnologias educacionais em sala de aula vêm se cercando de diversas experiências in loco, que, por vezes, reforçam a importância da prontidão dos professores em relação a aprender novas linguagens para tornar cada vez mais significativa a aprendizagem de conceitos importantes. No entanto, nenhuma dessas experiências - e talvez nem as vindouras - sejam suficientes para que a comunidade de educadores e estudiosos da educação atinja um consenso sobre como e porque utilizar ou deixar de utilizar essas ferramentas.

Busquei, neste trabalho, oferecer a professores do segundo ano do ensino médio mais uma utilização da ferramenta digital Geogebra para o ensino de física. O intuito é substanciar o argumento de que é necessário que a escola acompanhe as mudanças que as novas tecnologias promovem socialmente e, mais, que seja ela própria autora de mudanças sociais.

As tecnologias digitais são ferramentas interessantes e que a escola resiste em usar [19]. Entretanto, explorar as potencialidades educacionais dessas ferramentas de uma atitude meramente opcional, vem se convertendo em uma necessidade nas últimas décadas. Neste contexto, acredito que esse trabalho acrescenta uma abordagem interessante no ensino de óptica geométrica de lentes esféricas, ainda que minimamente testado.

Eu utilizo essa abordagem, quando possível, para ensinar esse tema para meus alunos do segundo ano do ensino médio. Apesar de simples, a mera utilização de uma ferramenta digital parece favorecer o engajamento de um número maior de alunos. Não foi possível verificar nessas aplicações se houve ganho de aprendizagem em relação à abordagem tradicional, pois não a aplicava com objetivo de recolher dados para uma pesquisa bem estruturada. Tudo que tenho são minhas impressões pessoais. Por isso mesmo, 
estou certo disso, essa proposta tem muito a melhorar.

Aconselho a quem interessar, a inserção dessa atividade em uma sequência didática estruturada sobre um objetivo claro, como uma UEPS [20] ou algo semelhante. Ao fazer isso, essa proposta deixa de ser material complementar para ser uma articuladora entre estágios de aprendizagem.

Alerto sobre o risco de incorrer na citada dicotomia quantitativo $\mathrm{x}$ qualitativo ao utilizar uma abordagem puramente descritiva para o ensino de conceitos de física em detrimento do fenômeno em si. Tendo isso em vista, sobre o tema óptica geométrica de lentes delgadas, há um utensílio muito comum em residências que pode servir de materialidade e motivação para esse tipo de descrição proposta neste trabalho: um visor para porta, ou, como é conhecido popularmente, olho mágico. A maior parte dos olhos mágicos são compostos por 4 lentes delgadas, não necessariamente esféricas, que compostas oferecem um comportamento óptico diferente dependendo do sentido de propagação da luz neste instrumento.

Por fim, devemos, nós educadores, estar conscientes de que não é exatamente a ferramenta que torna mais ou menos significativa a aprendizagem de nossos alunos, nem talvez seja essa nossa incumbência. Mas que é possível, lançando mão dos mais diversos recursos pedagógicos, estimular nossos alunos a aprender, cada um à sua maneira, os conceitos mais importantes em ciências.

\section{Referências}

[1] PIETROCOLA, Maurício. A matemática como estruturante do conhecimento físico. Caderno brasileiro de ensino de física 19.1 (2002): 93-114.

[2] GURGEL, Célia Margutti A. Educação para as ciências da natureza e matemáticas no Brasil: um estudo sobre os indicadores de qualidade do SPEC (1983-1997). Ciência \& Educação (Bauru) 8.2 (2002)

[3] PINHEIRO, Nilcéia Aparecida Maciel et al. Educação crítico-reflexiva para um ensino médio científico-tecnológico: a contribuição do enfoque CTS para o ensino aprendizagem do conhecimento matemático. (2005).

[4] RICARDO, Elio C.; FREIRE, Janaína CA. A concepção dos alunos sobre a física do ensino médio: um estudo exploratório. Revista Brasileira de Ensino de Fisica, v. 29, n. 2, p. 251-266, (2007).

[5] COELHO, André Luís Miranda de Barcellos. Aplicação do monocórdio e o uso de elementos musicais perceptuais como estruturantes para o ensino de conceitos da física ondulatória. 161 páginas. Dissertação de mestrado (2016).

[6] CARVALHO, Cristiane. Uma revisão de literatura sobre o uso de softwares/simuladores/applets e principais referenciais teóricos no ensino de física. Departamento de Ciências Naturais, Universidade Federal de São João del Rei, 2012.

[7] FILHO, Geraldo. Simuladores Computacionais para o ensino de física básica: uma discussão sobre produção e uso. Mestrado Profissional em Ensino de Física, Universidade do Rio de Janeiro, 2010. 
[8] YAMAMOTO, Issao; BARBETA, Vagner. Simulações de experiências como ferramenta de demonstração virtual em aulas de teoria de física. Revista Brasileira de Ensino de Física, vol. 23, no. 2, Junho, 2001

[9] ARANTES, Alessandra Riposati; MIRANDA, Márcio Santos; STUDART, Nelson. Objetos de Aprendizagem no ensino de física: usando simulações do PheT. Física na Escola, v. 11, n. 1, 2010.

[10] HEIDEMANN, Albuquerque; OLIVEIRA, Ângelo; VEIT, Eliane. Ferramentas online no ensino de ciências: uma proposta com o Google Docs. Universidade do Rio Grande Do Sul - Instituto de Física. Física na Escola, v.11, n.2, 2010.

[11] HECKLER, Valmir; SARAIVA, Maria; FILHO, Kepler. Uso de simuladores, imagens e animações como ferramentas auxiliares no ensino/aprendizagem de óptica. Revista Brasileira de Ensino de Física, v.29, n.2, p.267-273, 2007.

[12] Parâmetros Curriculares Nacionais. Secretaria de Educação Fundamental. Brasília: MEC/SEF, v. 1998, p. 2000, 1997.

[13] PISA, Brasil no PISA 2015 Análises e reflexões sobre o desempenho dos estudantes brasileiros. 2016

[14] DIKOVIĆ, Ljubica. Applications of GeoGebra into teaching some topics of mathematics at the college level. Computer Science and Information Systems, v. 6, n. 2, p. 191-203, 2009.

[15] NASCIMENTO, Eimard GA do. Avaliação do uso do software GeoGebra no ensino de geometria: reflexão da prática na escola. XII Encontro de Pós-Graduação e Pesquisa da Unifor, ISSN 8457 (1808): 2012.

[16] RIBEIRO, Tiago Nery; DO NASCIMENTO SOUZA, Divanízia. A utilização do software Geogebra como ferramenta pedagógica na construção de uma unidade de ensino potencialmente significativa (UEPS). Revista Sergipana de Matemática e Educação Matemática, v. 1, n. 1, p. 36-51, 2016.

[17] FEYNMAN, Richard P., R. B. Leighton, and M. Sands. Lições de Física, Vol. 1. Tradução de Adriana VR da Silva e KalineR. Coutinho. Porto Alegre: Bookman (2008).

[18] SILVA, Willians Gaviolida. O Geogebra como ferramenta de apoio à aprendizagem significativa em óptica geométrica. 2016. Dissertação de Mestrado. Universidade de São Paulo.

[19] LEVY, P.; As tecnologias da inteligência - o futuro do pensamento na era da informática. Lisboa, Instituto Piaget, 1994.

[20] MOREIRA, Marco Antonio. Unidades de enseñanza potencialmente significativas-UEPS. Aprendizagem Significativa em Revista/Meaningful Learning Review, Rio Grande do Sul 1.2, 2011. 